\title{
Interwell excitons in GaAs superlattices
}

Birkedal, Dan; Sayed, Karim El; Sanders, G.; Spiegelberg, C.; Vadim, Lyssenko; Stanton, C.; Hvam, Jørn Märcher; Timofeev, V. B.; Bayer, M.

Published in:

Physical Review B

Link to article, DOI:

10.1103/PhysRevB.54.10316

Publication date:

1996

Document Version

Publisher's PDF, also known as Version of record

Link back to DTU Orbit

Citation (APA):

Birkedal, D., Sayed, K. E., Sanders, G., Spiegelberg, C., Vadim, L., Stanton, C., Hvam, J. M., Timofeev, V. B., \& Bayer, M. (1996). Interwell excitons in GaAs superlattices. Physical Review B, 54(15), 10316-10319. https://doi.org/10.1103/PhysRevB.54.10316

\section{General rights}

Copyright and moral rights for the publications made accessible in the public portal are retained by the authors and/or other copyright owners and it is a condition of accessing publications that users recognise and abide by the legal requirements associated with these rights.

- Users may download and print one copy of any publication from the public portal for the purpose of private study or research.

- You may not further distribute the material or use it for any profit-making activity or commercial gain

- You may freely distribute the URL identifying the publication in the public portal 


\title{
Interwell excitons in GaAs superlattices
}

\author{
D. Birkedal, K. El Sayed, G. Sanders, ${ }^{*}$ C. Spiegelberg, V.G. Lyssenko, ${ }^{\dagger}$ \\ C. Stanton, ${ }^{*}$ and J. M. Hvam \\ Mikroelektronik Centret, The Technical University of Denmark, DK-2800 Lyngby, Denmark
}

V. B. Timofeev

Institute of Solid State Physics, Russian Academy of Sciences, 142432 Chenogolovka, Moscow district, Russia

\author{
M. Bayer \\ Technische Physik, Universität Würzburg, Am Hubland, D-97074 Würzburg, Germany
}

(Received 23 April 1996)

\begin{abstract}
The formation of spatially indirect excitons in superlattices with narrow minibands is theoretically and experimentally investigated. We identify the experimental conditions for the observation of interwell excitons and find a distinct excitonic state energetically located between the $1 \mathrm{~s}$ exciton and the onset of the miniband absorption. The interwell exciton is similar to the first Wannier-Stark localized exciton of an electrically biased superlattice. However, in the present case the localization is mediated by the Coulomb interaction of the electron and the hole without external fields. [S0163-1829(96)00739-4]
\end{abstract}

The excitonic properties of semiconductor superlattices have attracted considerable interest during the recent past. The gradual transition of the electronic and optical properties, from isolated quantum wells to those of a threedimensional solid for increasing miniband width, has been studied earlier experimentally ${ }^{1-3}$ and theoretically. ${ }^{1,4,5}$ These reports were focused on the observation of the predicted $M_{1}$ exciton associated with the upper miniband edge. The quality of the experimental data was recently disputed, ${ }^{6,7}$ and attempts were made using photocurrent and photoreflectance spectroscopy in addition to excitation spectroscopy. In these reports a feature associated with the lower miniband edge was resolved for some sample parameters but was not given any further attention.

By applying an electric field in the superlattice growth direction, the electron and hole wave functions localize to individual wells. This Wannier-Stark localization was observed experimentally by Mendez et al. ${ }^{8}$ and Voisin and co-workers ${ }^{9}$ leading to the experimental observations of Bloch oscillations by beats in the four-wave mixing signals ${ }^{10}$ and in the emission of electromagnetic radiation at the Bloch frequency. ${ }^{11}$ These phenomena are a consequence of the spatially indirect nature of the first Wannier-Stark localized state.

Similar effects have been studied in coupled double quantum wells. The resonant coupling of identical wells splits the exciton of the isolated wells into a symmetric/antisymmetric pair. Applying an electric field in the growth direction breaks the inversion symmetry of the structure and spatially indirect interwell excitons form. For increasing fields the symmetric/ antisymmetric splitting decreases and the double quantum well retains the properties of the single quantum well in the limiting case. ${ }^{12}$ Symmetric double quantum wells have also recently been studied in the presence of a magnetic field. ${ }^{13}$ It was found that a magnetic field perpendicular to the plane of the coupled quatum wells strongly modifies the exciton states and at a given symmetric/antisymmetric splitting transforms the system into the weak coupling regime.

In this paper, we demonstrate theoretically and experimentally that interwell excitons also can form in superlattices without externally breaking the inversion symmetry. For superlattices with narrow minibands the Coulomb interaction of electrons and holes in adjacent wells is sufficient to localize the superlattice state and form interwell excitons. The interwell exciton is observed directly in photoluminescence (PL) and absorption experiments. We confirm the $1 \mathrm{~s}$ in-plane character of the interwell exciton by recording its diamagnetic shift in photoluminescence excitation (PLE) spectra for varying magnetic fields in the growth direction.

The electronic coupling of states in a multiple quantum well structure forming a superlattice is characterized by a miniband width. A miniband is associated with both the conduction $(c)$ and the valence $(v)$ band states, and the combined miniband width, $\Delta=\Delta_{c}+\Delta_{v}$, is the sum of the conduction and the valence miniband widths. We have earlier argued $^{14}$ that a condition for the formation of interwell excitons is that the combined miniband width is smaller than the exciton binding energy of the isolated quantum well $\left(E_{b}^{2 \mathrm{D}}\right)$, and the interwell Coulomb energy $E_{\mathrm{IW}}=e^{2} / \epsilon d$, where $\epsilon$ is the dielectric constant, and $d$ is the superlattice period length, i.e., that

$$
\Delta<E_{\mathrm{IW}}<E_{b}^{2 \mathrm{D}} .
$$

For superlattices with narrow barriers both the interwell Coulomb energy and the combined miniband width is larger than the exciton binding energy of the isolated quantum well. For increasing barrier width the combined miniband width decreases exponentially. However, the interwell Coulomb energy decreases as $1 / d$ and at some barrier width we find that Eq. (1) is satisfied.

For superlattices with very narrow miniband widths one has to realize that the oscillator strength of the interwell ex- 
citon is very small and the effect is negligible. For an experimental observation, a superlattice sample has to be realized that satisfies Eq. (1) and have a miniband wide enough to give the interwell exciton sufficiently oscillator strength to be measured. We find that this criterion is met in a GaAs/ $/ \mathrm{Al}_{0.3} \mathrm{Ga}_{0.7} \mathrm{As}$ multiple quantum well structure with wells of $80 \AA$ and barriers of $50 \AA$. For these structural parameters, we find that $E_{b}^{2 \mathrm{D}} \approx 11.5 \mathrm{meV}$ and that the interwell Coulomb energy is estimated to $E_{\mathrm{IW}} \approx 8.5 \mathrm{meV}$. The combined miniband width of the corresponding superlattice is estimated, using a modified Kronig-Penney model, ${ }^{15}$ to be $2.6 \mathrm{meV}$.

For a calculation of the bound exciton states, we solve the $k$-space version of the exciton effective mass equation:

$$
\begin{aligned}
& {\left[E_{n}^{e}\left(\vec{k}_{\|}\right)+E_{m}^{h}\left(\vec{k}_{\|}\right)\right] G_{n, m}\left(\vec{k}_{\|}\right)} \\
& \quad+\sum_{n^{\prime}, m^{\prime}} \sum_{\vec{k}_{\|}^{\prime}} V_{n^{\prime}, m^{\prime}}^{n, m}\left(\vec{k}_{\|}, \vec{k}_{\|}^{\prime}\right) G_{n^{\prime}, m^{\prime}}\left(\vec{k}_{\|}^{\prime}\right)=E G_{n, m}\left(\vec{k}_{\|}\right),
\end{aligned}
$$

where $G_{n, m}\left(\vec{k}_{\|}\right)$is the exciton relative motion envelope function, and $n$ and $m$ run over conduction and valence band states, respectively. In the present calculation, we sum over the number of wells and neglect higher excited subbands. The Coulomb interaction term is given by

$$
\begin{aligned}
V_{n^{\prime}, m^{\prime}}^{n, m}\left(\vec{k}_{\|}, \vec{k}_{\|}^{\prime}\right)= & \frac{-e^{2}}{\epsilon\left|\vec{k}_{\|}-\vec{k}_{\|}^{\prime}\right|} \iint f_{n^{\prime}}\left(z_{c}\right) f_{n}\left(z_{c}\right) f_{m^{\prime}}\left(z_{v}\right) \\
& \times f_{m}\left(z_{v}\right) \exp \left(-\left|\vec{k}_{\|}-\vec{k}_{\|}^{\prime}\right|\left|z_{c}-z_{v}\right|\right) d z_{c} d z_{v},
\end{aligned}
$$

where $f_{n}\left(z_{c}\right)$ and $f_{m}\left(z_{v}\right)$ are the conduction band and valence band envelope functions, respectively. The exciton wave function is written as

$$
\psi_{x}\left(\vec{r}_{\|}, z_{c}, z_{v}\right)=\sum_{n, m} \sum_{k_{\|}} G_{n, m}\left(\vec{k}_{\|}\right) f_{n}\left(z_{c}\right) u_{c}(\vec{r}) f_{m}\left(z_{v}\right) u_{v}(\vec{r})
$$

where $u_{c, v}(\vec{r})$ are the periodic parts of the Bloch functions for the conduction and valence bands, respectively. In the present calculation, the effects of band mixing are neglected and only heavy hole states are included. The presented data are for a calculation including five wells. The solution of Eq. (2) gives not only the ground state exciton but also the excited state excitons and their envelope functions. The five lowest lying solutions to Eq. (2) are almost degenerate and correspond to the intrawell excitons of the isolated quantum wells. We also find eigenvalues of Eq. (2) $7 \mathrm{meV}$ above the lowest lying states with substantial oscillator strength ( $\approx 10 \%$ of the intrawell excitons). These states are of $1 s$ character, i.e., without nodes in the in-plane envelope function. The square of the exciton envelope function for such a state is shown in Fig. 1, where $\left|\psi_{x}\left(z_{c}, z_{v}\right)\right|^{2}$ represents the probability of finding the electron at $z_{c}$ and the hole at $z_{v}$. It is clear from the figure that the electron and the hole parts of the exciton are confined to different wells, i.e., we are dealing with interwell excitons. However, note that the solution

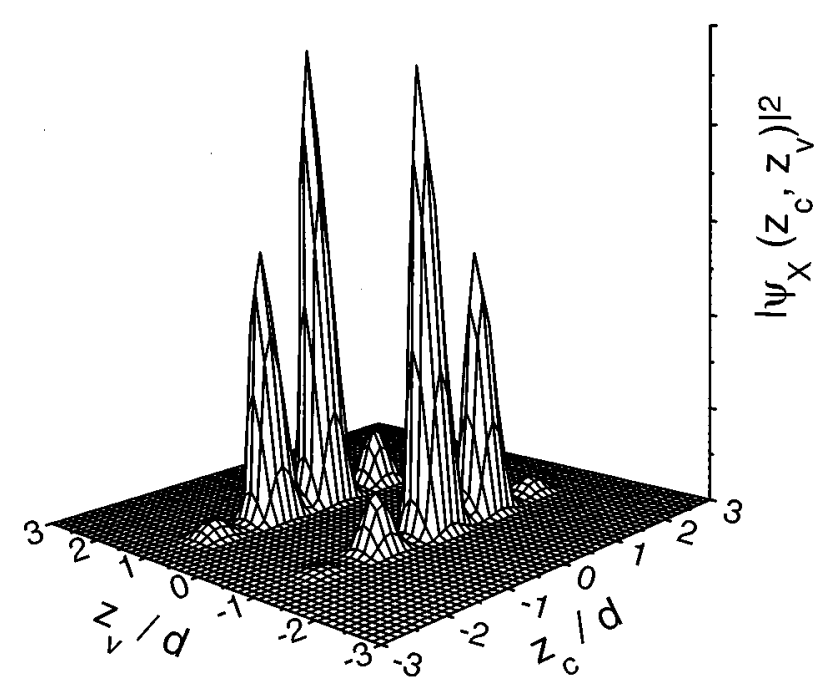

FIG. 1. Exciton envelope function for the interwell exciton in a GaAs- $\mathrm{Al}_{0.3} \mathrm{Ga}_{0.7} \mathrm{As}$ multiple quantum well with $80 \AA$ wells and 50 $\AA$ barriers.

consists of two degenerate pairs of opposite symmetry restoring the total inversion symmetry of the structure.

A sample structure was grown by molecular beam epitaxy on a semi-insulating GaAs substrate, and was composed of 20 wells of $80 \AA \mathrm{GaAs}$ separated by $50 \AA$ barriers of $\mathrm{Al}_{0.3} \mathrm{Ga}_{0.7} \mathrm{As}$. Low-temperature PL experiments were performed in a continuous-flow $\mathrm{He}$ cryostat held at $4 \mathrm{~K}$. The luminescence was excited by the $632.8 \mathrm{~nm}$ line of a $\mathrm{HeNe}$ laser and the signal was recorded using a photon counting system and a cooled GaAs-cathode-photomultiplier tube. The PLE experiments were conducted in a He cryostat with a superconducting solenoid for a variable magnetic field up to $13 \mathrm{~T}$, using a tunable $\mathrm{cw}$ Ti:sapphire laser as an excitation source. In the PLE experiments a magnetic field parallel to the superlattice direction was used. For transmission experiments samples were lifted off the substrate and mounted on a sapphire disk. The transmission spectra were recorded by an optical multichannel analyzer using white light from a tungsten halogen lamp.

The PL and absorption spectra for the sample are shown in Fig. 2. Heavy and light hole exciton absorption peaks are easily identified in the absorption spectrum. In addition, a clear absorption peak $6.8 \mathrm{meV}$ above the heavy hole exciton line is seen in the spectrum. A feature $\left(I_{\mathrm{HH}}\right)$ is seen in the PL data coinciding with the peak seen in the absorption spectrum at the high energy side of the main heavy hole exciton luminescence line $\left(D_{\mathrm{HH}}\right)$. Luminescence from the light hole exciton was only observed at higher temperature or at the highest excitation densities. The shoulder on the low energy side of the $D_{\mathrm{HH}}$ line is due to excitons localized on impurities and defects.

The strength of the $\mathrm{I}_{\mathrm{HH}}$ feature in both the absorption and luminescence experiment indicate that the feature is not related to the usual $2 s$ state of the exciton seen in isolated quantum wells. Comparing the luminescence and absorption data also shows that the $I_{\mathrm{HH}}$ feature is not in thermal equilibrium with the $1 s$ state of the heavy hole exciton $(1 s \mathrm{HH})$ 


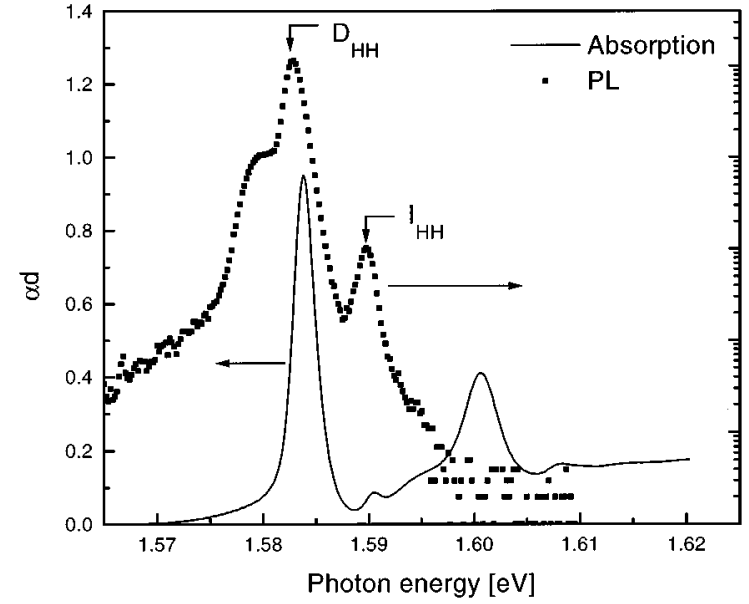

FIG. 2. Photoluminescence and absorption spectra of the sample at $5 \mathrm{~K}$.

as expected for the $2 s \mathrm{HH}$ state. In fact, we observe a much higher population of the $\mathrm{I}_{\mathrm{HH}}$ state than expected from thermal population from the $1 s \mathrm{HH}$ exciton, indicating a slow transfer of carriers between the two states. This is the expected behavior of interwell excitons since they can only relax to the intrawell exciton state by a phonon assisted tunneling process. ${ }^{16}$

For further confirmation that the $I_{\mathrm{HH}}$ feature is indeed the interwell exciton, we have performed PLE measurements in magnetic fields. In the weak field limit, where the cyclotron energy is much less than the exciton binding energy, the diamagnetic shift of an exciton is proportional to the square

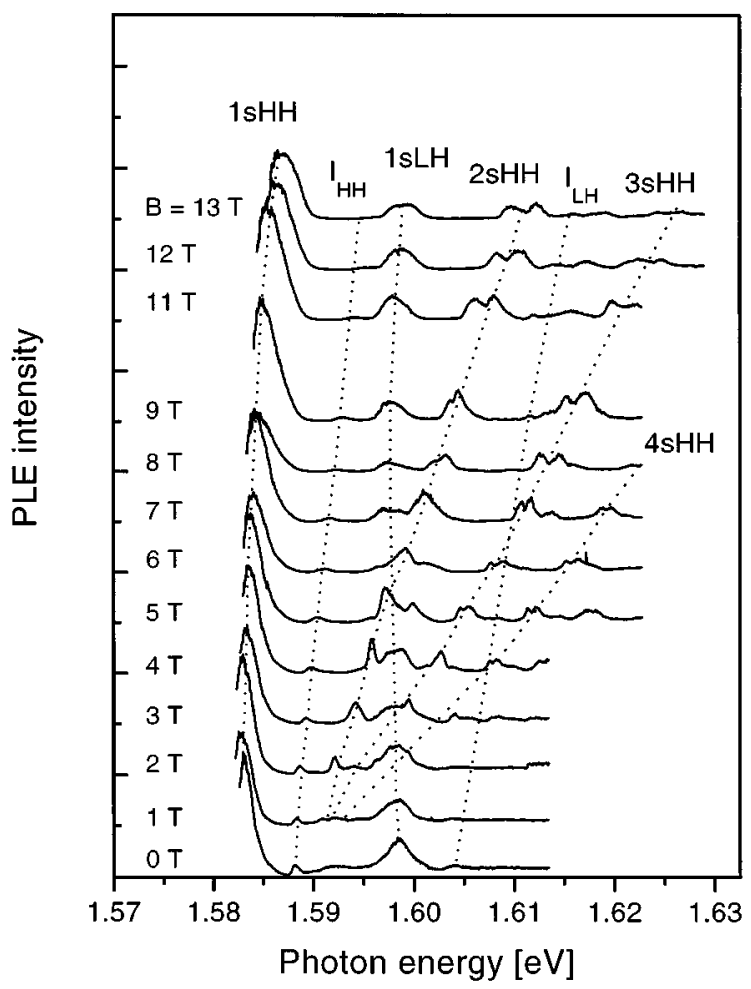

FIG. 3. Photoluminescence excitation spectroscopy at different magnetic fields.

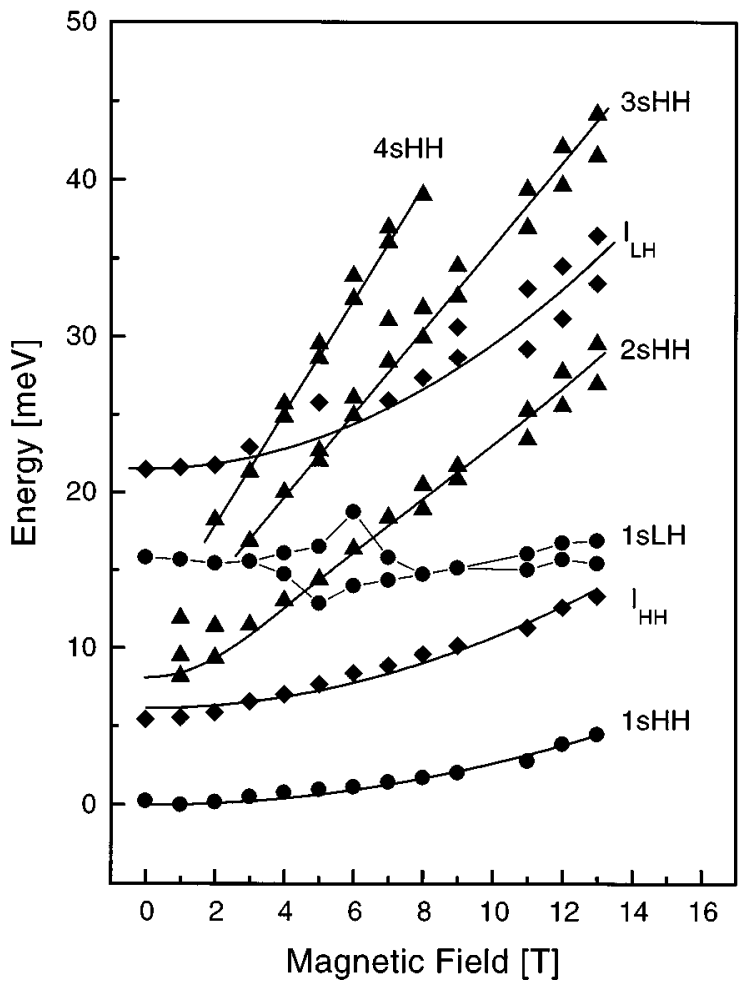

FIG. 4. Exciton peak positions as a function of magnetic field.

of the exciton radius and inversely proportional with the exciton binding energy. The radii of an exciton series in two dimensions can be expressed as $a_{n}=a_{0}(2 n+1)$, where $n=0,1, \ldots$ is the exciton quantization number. ${ }^{17}$ Hence, the ratio of the diamagnetic shifts of the $1 s$ and the $2 s$ excitons equals 9 . The interwell exciton has approximately half the binding energy of the $1 s \mathrm{HH}$ exciton and we would expect to find a ratio of the interwell and $1 s \mathrm{HH}$ diamagnetic shifts near 2.

Figure 3 shows PLE spectra from the sample for different magnetic fields. The $1 s \mathrm{HH}$ and $1 s \mathrm{LH}$ excitons show large oscillator strengths and can easily be identified. With increasing magnetic field they exhibit a diamagnetic shift, which is larger for the heavy hole than for the light hole exciton because of the larger binding energy of the light hole exciton. In fact, we observe a near zero diamagnetic shift of the $1 s \mathrm{LH}$ exciton, which we attribute to a stronger superlattice effect on the light hole states. This is similar to the observation by Butov et al. ${ }^{13}$ where, in strongly coupled quantum wells, the magnetic field transformed the system into the weak coupling regime.

In addition to the ground state excitons, which transform with increasing magnetic field into the lowest Landau levels $(N=0)$, a large number of transitions is observed which can be attributed to higher Landau levels $(N>1)$ originating from excited excitonic states at $B=0$. Only excitons composed of electrons and holes with the same Landau level quantum number $N$ are optically active. In Fig. 3, the $2 s \mathrm{HH}, 3 s \mathrm{HH}$, and $4 s \mathrm{HH}$ magnetoexcitons can be clearly identified. The $2 s \mathrm{HH}$ state can be resolved even at small magnetic fields around $1 \mathrm{~T}$. For increasing magnetic field this magnetoexciton state crosses the $1 s \mathrm{LH}$ state, which is allowed due to their different symmetry character. However, 
in the region of crossing of these states a redistribution of the oscillator strengths is observed due to $\mathrm{HH}-\mathrm{LH}$ mixing. ${ }^{13}$

The $\mathrm{I}_{\mathrm{HH}}$ state observed in the PL and absorption spectra is clearly resolved in the PLE spectra. This state demonstrates a stronger diamagnetic shift than the $1 s \mathrm{HH}$ exciton, and based on the magneto PLE data, we will now prove that the $I_{\mathrm{HH}}$ state is indeed the interwell exciton. The shifts of the different excitonic states with magnetic field are shown in Fig. 4. By fitting a quadratic expression to the data in Fig. 4, we find the diamagnetic shift of the $1 \mathrm{sHH}$ exciton to be 26.4 $\mu \mathrm{eV} / \mathrm{T}^{2}$, in good agreement with earlier published values $\left(29 \mu \mathrm{eV} / \mathrm{T}^{2}\right.$ for a $75 \AA$ well $) .{ }^{18}$ A larger diamagnetic shift of $45.3 \mu \mathrm{eV} / \mathrm{T}^{2}$ is found for the $I_{\mathrm{HH}}$ exciton. The ratio of the diamagnetic shifts of the $I_{\mathrm{HH}}$ and the $1 s \mathrm{HH}$ is thus 1.85 , clearly showing that the $I_{\mathrm{HH}}$ state is unrelated with the $2 s \mathrm{HH}$ exciton, and in full accord with the interwell exciton of the heavy hole.

We determine the $2 s-1 s$ splitting at $B=0$ by extrapolating the $2 s$ transition energies for $B \rightarrow 0$ by eye. The exciton binding energy can therefore be estimated by multiplying this splitting by a factor of $9 / 8$, which is valid for a ideal two-dimensional exciton. ${ }^{17}$ Thus, we find a binding energy of the $1 s \mathrm{HH}$ exciton of $\approx 9 \mathrm{meV}$. This value is slightly smaller than what we find for an isolated $80 \AA$ quantum well $(11.5 \mathrm{meV})$ and is related to the superlattice effect. Furthermore, we resolve a splitting of the $2 s \mathrm{HH}$ and $2 s \mathrm{LH}$ states into doublets. The splitting in energy of these doublets increases linearly with $B$, and can therefore be attributed to spin splitting. We also observed peaks in the PLE data of Fig. 3 which have been labeled $I_{\mathrm{LH}}$ in Fig. 4. This peak shows a diamagnetic shift, which is found to be 80 $\mu \mathrm{eV} / \mathrm{T}^{2}$. The $I_{\mathrm{LH}}$ peak extrapolates at zero magnetic field to the continuum edge of the light hole state and is therefore assigned to the light hole interwell exciton.

In summary, we have theoretically and experimentally shown the presence of a distinct excitonic state above the $1 \mathrm{~s}$ exciton of a superlattice, which is spatially indirect in nature. The state is similar to the interwell states of the WannierStark ladder. However, in the present case the Coulomb forces alone are responsible for the localization of the state in the absence of an applied electric field. These results provide a fundamental insight into the optical properties of superlattices and are expected to be important in the interpretation of recent four-wave mixing experiments investigating Bloch oscillation from superlattices.

We would like to thank C.B. Sørensen for the sample preparation and $\mathrm{W}$. Langbein for many useful discussions. This work was supported by the Danish Natural Science Research Council, The Danish Research Academy, and the Danish Center for Nanostructures - CNAST. V.B.T. acknowledges financial support for this work from the Alexander von Humboldt Foundation and the Russian Science Foundation.
*Permanent address: Department of Physics, University of Florida, Gainesville, FL.

${ }^{\dagger}$ Permanent address: Institute of Microelectronics Technology, and Superpure Materials, Chernogolovka, Russia.

${ }^{1}$ A. Chomette, B. Lambert, B. Deveaud, F. Clérot, A. Regreny, and G. Bastard, Europhys. Lett. 4, 461 (1987).

${ }^{2}$ J.J. Song, P.S. Jung, Y.S. Yoon, H. Chu, Y.-C. Chang, and C.W. Tu, Phys. Rev. B 39, 5562 (1989).

${ }^{3}$ B. Deveaud, A. Chomette, F. Clérot, A. Regreny, J.C. Maan, R. Romestain, and G. Bastard, Phys. Rev. B 40, 5802 (1989).

${ }^{4}$ H. Chu and Y.-C. Chang, Phys. Rev. B 36, 2946 (1987).

${ }^{5}$ H. Chu and Y.-C. Chang, Phys. Rev. B 39, 10861 (1989).

${ }^{6}$ K. Fujiwara, K. Kawashima, T. Yamamoto, N. Sano, R. Cingolani, H.T. Grahn, and K. Ploog, Phys. Rev. B 49, 1809 (1994).

${ }^{7}$ K. Fujiwara, K. Kawashima, T. Yamamoto, and K. Ploog, Solid State Electron. 37, 889 (1994).

${ }^{8}$ E.E. Mendez, F. Agullò-Rueda, and J.M. Hong, Phys. Rev. Lett. 60, 2426 (1988).
${ }^{9}$ P. Voisin, J. Bleuse, C. Bouche, S. Gaillard, C. Alibert, and A. Regreny, Phys. Rev. Lett. 61, 1639 (1988).

${ }^{10}$ K. Leo, P. Bolivar, F. Brüggemann, and R. Schwedler, Solid State Commun. 84, 943 (1992).

${ }^{11}$ H.G. Roskos, M.C. Nuss, J. Shah, K. Leo, D.A.B. Miller, A.M. Fox, S. Schmitt-Rink, and K. Köhler, Phys. Rev. Lett. 68, 2216 (1992).

${ }^{12}$ G. Bryant, Phys. Rev. B 47, 1683 (1993).

${ }^{13}$ L.V. Butov, A. Zrenner, G. Abstreiter, A.V. Petinova, and K. Eberl, Phys. Rev. B 52, 12153 (1995).

${ }^{14}$ D. Birkedal, K. El Sayed, G. Sanders, V.G. Lyssenko, C. Stanton, and J.M. Hvam, Il Nuovo Cimento 17D, 1359 (1995).

${ }^{15}$ G. Bastard, Wave Mechanics Applied to Semiconductor Heterostructures (Les editions de Physique, Les Ulis, France, 1988).

${ }^{16}$ B. Deveaud, F. Clerot, A. Chomette, A. Regreny, R. Ferreira, G. Bastard, and B. Sermage, Europhys. Lett. 11, 367 (1990).

${ }^{17}$ M. Shinada and S. Sugano, J. Phys. Soc. Jpn. 21, 1936 (1966).

${ }^{18}$ D.C. Rogers, J. Singleton, R.J. Nicholas, C.T. Foxon, and K. Woodbridge, Phys. Rev. B 34, 4002 (1986). 